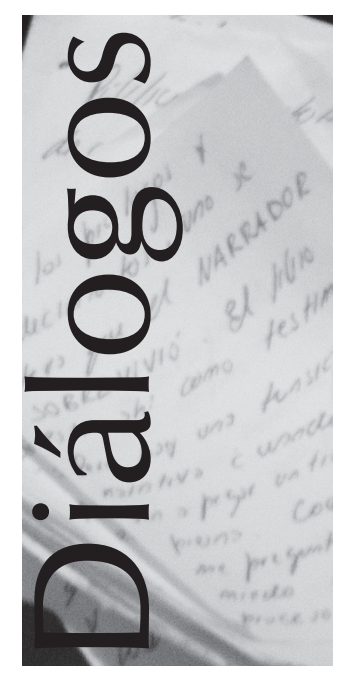

\title{
Práctica editorial contextualizada: Maria Cecília de Souza Minayo y la revista Ciência \& Saúde Coletiva
}

\author{
Contextualized editorial practices: Maria \\ Cecília de Souza Minayo and the journal \\ Ciência \& Saúde Coletiva
}

Hugo Spinelli ${ }^{1}$, Viviana Martinovich ${ }^{2}$

RESUMEN Esta entrevista forma parte de una serie de diálogos que Salud Colectiva viene organizando con diversos editores de referencia, que tienen como propósito abordar las dificultades similares por las que atraviesan las revistas latinoamericanas y las diversas formas de resolver esas dificultades, las cuales pueden ser un punto de partida para encontrar soluciones contextualizadas. En esta entrevista realizada en 2015, en el Instituto de Salud Colectiva de la Universidad Nacional de Lanús, Maria Cecília de Souza Minayo nos habla del contexto de surgimiento de la revista Ciência \& Saúde Coletiva, de las disputas políticas dentro del campo de la salud colectiva, y de la relevancia del debate como proceso de constitución de la propia ciencia. Además, analiza la importancia del financiamiento para el crecimiento de una revista, y los problemas y las dificultades para obtenerlo, lo que expresaría la falta de una política brasileña de publicación científica.

PALABRAS CLAVES Políticas Editoriales; Edición; Sistemas de Evaluación de las Publicaciones; Brasil.

ABSTRACT This interview forms part of a series of dialogues that Salud Colectiva has been holding with key editors in the field, with the purpose of examining the similar difficulties confronted by Latin American journals and the diverse ways the journals have faced these difficulties as a likely starting point for uncovering contextualized solutions. In this interview, carried out in 2015 in the Instituto de Salud Colectiva of the Universidad Nacional de Lanús, Maria Cecília de Souza Minayo speaks to us about the context in which the journal Ciência \& Saúde Coletiva emerged, the political disputes existing within the collective health field, and the relevance of debate as a constitutive process of science itself. Additionally, Minayo analyzes both the importance of funding to the growth of a journal and the problems and difficulties in obtaining funds, which puts into evidence the lack of policy regarding scientific publishing in Brazil.

KEY WORDS Editorial Policies; Publishing; Systems for Evaluation of Publications; Brazil.

'Doctor en Salud Colectiva. Director del Instituto de Salud Colectiva, Universidad Nacional de Lanús, Buenos Aires, Argentina. $\square$ (iD)

${ }^{2}$ Editora ejecutiva. Instituto de Salud Colectiva, Universidad Nacional de Lanús, Buenos Aires, Argentina. $\square$ 


\section{INTRODUCCIÓN}

Maria Cecília de Souza Minayo se graduó en Sociología en la Universidade Federal do Rio de Janeiro, y en Ciencias Sociales, en la City University of New York. Es magíster en Antropología Social por la Universidade Federal do Rio de Janeiro y doctora en Salud Pública por la Fundação Oswaldo Cruz. Desde 1997 es editora científica de la revista Ciência \& Saúde Coletiva de la Associação Brasileira de Saúde Coletiva (ABRASCO) e investigadora titular de la Fundação Oswaldo Cruz.

Es referente en el área de la investigación cualitativa, con obras ya clásicas como $E I$ desafío del conocimiento: investigación cualitativa en salud, con varias ediciones en portugués y español, y reeditada en 2009 en una versión ampliada bajo el título La artesanía de la investigación cualitativa; y en temas como violencia y salud, en los que ha coordinado una gran cantidad de proyectos, líneas de investigación y publicaciones desde el Centro Latino-Americano de Estudos de Violência e Saúde "Jorge Careli" (CLAVES), del cual es directora desde su creación en 1988 hasta la actualidad.

Ha recibido varios premios, entre ellos, la Medalha de Mérito da Saúde "Oswaldo Cruz" otorgado en 2009 por el Ministerio de Salud y el Prêmio de Direitos Humanos, otorgado por la Presidencia de la Republica de Brasil en 2014.

Para los quetrabajamos en Salud Colectiva, Maria Cecília es una gran editora pero, sobre todo, una madrina, una "terapeuta" a la que recurrimos ante situaciones problemáticas que, especialmente en los primeros años de la revista, resultaban de difícil resolución, y siempre nos brindó su experiencia y apoyo. Para el Instituto de Salud Colectiva (ISCo) de la Universidad Nacional de Lanús, es una referente teórico-metodológica de una generosidad sin límites, que permitió la creación de la sede argentina del CLAVES en el ISCo, así como el intercambio de estudiantes y docentes, lo cual generó un vínculo afectivo muy fuerte entre ambos equipos.
Fue invitada en 2015 para la apertura de la decimoctava cohorte de la Maestría en Epidemiología, Gestión y Políticas de Salud del ISCo, y aprovechamos su visita para dialogar sobre su experiencia como editora de la revista Ciência \& Saúde Coletiva, una de las revistas más importantes del área de salud colectiva de la región, que recibe más de 2.400 artículos por año y publica un promedio de 350 artículos en sus 12 números anuales.

\section{DIÁLOGO}

Hugo Spinelli: Para nosotros fue muy difícil llegar a la situación actual en la que se encuentra nuestra revista, y lo logramos básicamente hablando con vos, que nos ayudaste mucho. Lo que notamos es que hay un vacío en cuanto a cómo se hace una revista, y ese vacío genera cierta confusión. Por ejemplo, un cardiólogo, porque es bueno en su especialidad, cree que puede hacer una buena revista, y la experiencia muestra que no es así. Creo que es un problema de la mayoría de los países de América Latina, excepto Brasil. La idea, entonces, es que a través de estas entrevistas se pueda mostrar cuáles son los problemas que se presentan en ese esfuerzo por hacer una buena revista. Carlos Coimbra Jr. publicó una serie de editoriales y notas en esta línea en Cadernos de Saúde Pública, eran textos muy útiles, que nosotros recuperamos.

Maria Cecília de Souza Minayo: Carlos Coimbra nos ayudó mucho desde el inicio, es una persona muy generosa. Cuando él asumió como editor científico de Cadernos de Saúde Pública se encontró con una revista parroquial, absolutamente parroquial, no tenía normas. Yo formaba parte del comité editorial, y era también... una parroquiana [risas...]. Y Coimbra comenzó a estudiar e introdujo las normas del grupo Vancouver en las referencias de la revista. En Vancouver, todos los años se reúnen editores científicos para discutir diversos temas vinculados a la calidad y la eficacia del proceso editorial. 
Pero por aquel entonces era un salto muy grande para nosotros discutir los temas del grupo Vancouver porque estaban más vinculados a la visibilidad internacional de la revista que a los primeros pasos que comenzábamos a transitar.

Yo empecé como editora -porque estoy como editora desde el inicio- con todas las dificultades, como cualquier otro editor. Pero me serví de la ayuda de Coimbra, quien me acercó algunas cosas para leer y siempre me dio una mano cuando necesité de su experiencia.

Viviana Martinovich: ¿Cómo y por qué surge Ciência \& Saúde Coletiva?

MCSM: La revista surgió justo después de la $X$ Conferencia Nacional de Salud. En esa época yo era presidenta de ABRASCO y bajo mi gestión hicimos dos grandes congresos. Por un lado, el I Congreso de Ciencias Sociales y Salud y, por otro, un Congreso Internacional de Epidemiología que, si no recuerdo mal, era el tercero. Ese congreso se realizó en Bahía y trajo a los mejores epidemiólogos del mundo. El comité de epidemiología de ABRASCO era y es de altísimo nivel, muy bien estructurado y consiguió mucho dinero para ese congreso, sobre todo del Ministerio de Salud. Entonces, lograron ciertas ventajas dentro del área de salud colectiva, en la que siempre existía una lucha con relación a la independencia de la epidemiología respecto de la salud colectiva, porque se decía que la epidemiología podía caminar sola... Había un discurso de grandeza y de hegemonía de la epidemiología dentro del área de salud colectiva. Y es interesante, porque cuando leés trabajos de grandes epidemiólogos brasileños, en algunos aparece la epidemiología como la propia salud colectiva, como la salud pública. En otros, ellos plantean la epidemiología como un instrumento de la salud pública. Es muy interesante leer esos textos de forma crítica, desde un punto de vista académico. Y en la última reunión antes de que yo deje la presidencia de ABRASCO, hicimos una reflexión muy profunda sobre qué significaría que la epidemiología se independizara del campo de la salud colectiva. Nosotros no queríamos eso, nadie lo quería, las grandes cabezas de la epidemiología también no querían eso, pero había un movimiento interno que sí lo planteaba. Y, en ese marco, surgió la idea de crear una revista brasileña de epidemiología y se produjo un impasse: ¿cómo crear una revista brasileña de epidemiología sin tener una revista de salud colectiva, generosa, que abarque el campo y las intersecciones? Entonces, esa reunión fue fundamental, porque llegamos a la conclusión de que nosotros no podíamos prohibir que el área de epidemiología creara una revista, pero teníamos que crear una revista de salud colectiva antes que el área de epidemiología creara la suya. Era una cuestión casi de lealtad con el campo. El campo no era solo de epidemiología, y aún hoy les digo a los colegas de epidemiología que su área sigue siendo la más fuerte, no cabe duda de que, de todas las áreas de la salud colectiva, es la más fuerte, pero no es la única y eso es necesario explicitarlo.

Ahí comenzamos a pensar cómo crear la revista, quién la dirigiría, etc. En aquella época, yo había sugerido como editor a una persona que no sé si ustedes conocen, que es Francisco Inácio Pinkusfeld Monteiro Bastos. Es epidemiólogo, fui su tutora y es una persona fantástica, un gran cientista, reconocido internacionalmente, con la cabeza muy abierta, y todo el mundo dijo: "él puede ser lo que sea pero su nombre no es reconocido en el campo. Tenemos que crear la revista con una persona que tenga un nombre que todos respeten". Y la dirección de ABRASCO me asignó esa responsabilidad. Yo me estaba yendo de la presidencia de ABRASCO y entonces lo acepté como un compromiso dentro de ese contexto.

A comienzos de 1996 empezamos a discutir el nacimiento de la revista y fue complicado porque en las reuniones muchas personas creían que estábamos entrando en una era digital y que una revista en papel no tendría futuro. Pero luego de una larga discusión se llegó a la conclusión de que debíamos crear una revista y tenía que ser impresa, 
y mi opinión en aquel momento era que todo lo que estaba viendo y que continúo viendo hasta hoy es una convergencia de soportes. Por ejemplo, hay muchos periódicos y revistas que disminuyen la cantidad de ejemplares impresos, crean otros mecanismos, le dan otra función y sostienen ambas versiones. $Y$ eso se fue transformando en un consenso, del mismo modo que la creación del nombre. Yo quería colocar "ciencia" porque en el contexto en el que estábamos, tal como menciona Bourdieu, la propia conformación del campo era una lucha política, entonces el término "ciencia" tenía su peso, es decir, no estoy hablando de cualquier cosa, estoy hablando de ciencia, la salud colectiva es una ciencia que trabaja con determinados conceptos.

A fines de 1996 salió la primera edición. Era fea, fea [risas...]. Ese primer número lo hicimos Peco [Péricles Silveira da Costa] y yo, los dos intentamos hacer todo. Para ese primer número invitamos a una serie de personas $y$, a pesar de que era fea, teníamos una idea que conservamos hasta hace muy poco tiempo, que era que el primer artículo de la revista siempre fuera un debate, lo cual se basaba en la concepción de que la ciencia es abierta, la vas construyendo, la vas corrigiendo, tenés controversias, y aún más en el área de la salud. En aquella época, la revista Veja publicaba un debate con páginas amarillas y nosotros la imitamos y pusimos las páginas amarillas [risas...]. Entonces, invitamos a distintas personas y publicamos creo que ocho artículos. Hoy todo ese acervo esta digitalizado y publicado en la página web de la revista $^{(1)}$ y en la biblioteca SciELO ${ }^{(2)}$.

Habíamos decidido que la revista fuera semestral. Pero el segundo año, ya en 1997, fue muy difícil y como Peco y yo éramos los que decidíamos todo, Peco resolvió publicar un solo ejemplar que reuniera los dos números del año. Esa edición es muy buena, incluyó una discusión sobre las políticas de salud, y un artículo de Naomar ${ }^{[a]}$ muy citado hasta hoy, que es una reflexión sobre métodología y transdisciplinariedad ${ }^{(3)}$.

Cuando asumí la gestión de ABRASCO, el área de salud colectiva en la Coordenação de Aperfeiçoamento de Pessoal de Nivel Superior (CAPES) y en el Conselho Nacional de Desenvolvimento Científico e Tecnológico (CNPq) estaba absolutamente desacreditada, había una disputa con el área de medicina, a tal punto que colocaron en los comités de CNPq y CAPES que nos evaluaban, a personas que no eran de nuestra área. Mi antecesor, Arlindo Gómez de Sousa, me llamó y me dijo: "Creo que vamos a tener que dar una respuesta a CAPES y a CNPq, particularmente a CNPq", y yo sostuve que dar una respuesta no era hacer una carta política, sino que era hacer un estudio sobre el área, una evaluación del área. $Y$ así lo hicimos: evaluamos los posgrados y publicamos los resultados de esa evaluación en la revista, recién creada.

Entonces, en 1998, para el tercer año de la revista, esa evaluación mostró que la salud colectiva tenía los mismos problemas que todas las otras áreas, tenía algunas ventajas y algunas dificultades. Convocamos a consultores internacionales para que colaboraran con nosotros en la etapa final, hicimos un seminario, y la revista publicó esa discusión.

$Y$ después de ese ejemplar, yo miraba esa revista fea y pensaba: "esto no va a ir lejos, no". Porque cuando las personas la agarran y ven ese papel feo [risas...] no les da ganas de leerla [risas...]. Ahí hablé con Peco y le dije: "Peco, o nosotros avanzamos o yo paro aquí, porque no voy a estar llevando esta cosa pequeña, pobre..." [risas...]. En aquella época teníamos dinero y consultamos a Coimbra, él había contratado a un diseñador para hacer el diseño de Cadernos de Saúde Pública y, entonces, nosotros contratamos a la misma persona. No solo la contratamos como diseñador, sino también para los servicios de edición, y nos cobraba muchísimo dinero.

Para el segundo número de 1998 la revista ya era bonita, ya era el modelo que tenemos hasta hoy. Para no encarecerla demasiado desde el punto de vista del diseño, incorporamos la figura que está en la tapa, no sé si se percibe, pero es un trabajo de diseño sobre una foto tomada desde un edificio de la avenida Río Branco, en la que se ve gente transitando en la calle, que representa esa 
idea de lo colectivo. A partir de 1998 empezamos a recibir artículos de forma espontánea, porque en los números iniciales todos los artículos llegaban por invitación. Siempre leí mucho sobre publicación científica y le consultaba a Coimbra, que también me daba material, mandaba textos, y fuimos trabajando y mejorando.

En la fiesta de los 20 años estaba la muchacha que fue la primera secretaria de la revista, que conseguimos financiar a través de la Fiocruz, de la "madre" Fiocruz, que sigue siendo "madre y padre" de la revista, porque se hace cargo de muchas cosas. ABRASCO es, como decimos nosotros, "pequeñas empresas y grandes negocios", hace millones de cosas pero el financiamiento que puede aportar es insignificante. Todo es de ABRASCO, pero el financiamiento de la revista tengo que buscarlo en $\mathrm{CNPq}$, en los propios autores que organizan números temáticos y consiguen financiamiento, en el Ministerio de Salud, en la Fundação de Amparo à Pesquisa do Estado do Rio de Janeiro (FAPERJ), o sea, tengo que buscar el financiamiento por fuera. Nunca en estos 20 años pude depender de los fondos de ABRASCO, y esa fragilidad financiera continúa hasta hoy.

Hay algunas fechas que creo que son centrales para la revista. Si no me equivoco, en el año 1998 comenzó SciELO Brasil y las revistas que entraron fueron las revistas consolidadas, como Cadernos de Saúde Pública -Coimbra formó parte del primer comité consultivo de SciELO-, Revista de Saúde Pública, y obviamente la nuestra no. Pero yo comencé a luchar para entrar, y en 2002 lo conseguí. La evaluación de SciELO fue muy positiva, pero ellos exigían que la revista publicara como mínimo tres números al año. Ahí decidimos pasar directamente a cuatro números por año, porque teníamos material para publicar, solo necesitábamos financiamiento.

Entonces, en 2002 pasamos a publicar cuatro números por año y comencé a luchar para ingresar a Medline. En 2005, ingresamos a Medline y en 2006 pasamos a publicar seis números por año porque la demanda era creciente. En 2007 ingresamos a Web of
Science y Scopus, que son solo algunas de las 23 bases de datos en las que estamos actualmente. En 2011 pasamos a ser una revista mensual, porque podés rechazar artículos, pero no podés rechazar el $99 \%$, es imposible. En aquel momento, ABRASCO le pide a Luiz Augusto Facchini, que por entonces era presidente de la asociación, que hagamos una revista mensual de 50 artículos por número, a lo que respondí: "Ios artículos los tenemos, pero quién lo va a financiar", porque es muy sencillo decir eso.

VM: Ante ese aumento de la periodicidad, ¿Cómo fue cambiando el equipo de trabajo?, ¿cómo fueron dando respuesta a las excesivas demandas de trabajo?

MCSM: La primera secretaria que tuvimos, Lilia, trabajó hasta 2004 y después se retiró por razones personales. Mientras ella estaba, habíamos conseguido que ABRASCO financiara a una pasante. Cuando se fue Lilia, publicábamos seis números por año, y ahí planteé que no podíamos continuar con ese equipo y ABRASCO contrató a tres personas, una que ocupaba el lugar de Lilia, otra para la comunicación con los autores -recibimos 2.400 originales por año por lo que se necesita una persona abocada a esa tarea-, y una bibliotecaria que era pasante y que fue quedándose... Les pagaban muy mal, y les siguen pagando mal hasta hoy. Y ahí dije: "se necesita una persona más" y explicité todas las actividades que necesitaba que hiciera esa persona: prefería que fuera periodista, capaz de hacer una gacetilla de prensa, que entendiera inglés y pudiera comunicarse con los autores que mandan los trabajos en inglés, etc.

Entonces, en realidad, trabajando cotidianamente, todo el tiempo, tenemos a cuatro personas: una periodista, una bibliotecaria, una persona dedicada a la comunicación con los autores en los procesos de revisión, y otra persona dedicada a la articulación con la parte de edición. En cuanto a la edición, conseguí que Fiocruz pagara la diagramación, a las personas que realizan la corrección de estilo en portugués y en inglés, 
y a la imprenta. Lo ideal para mí, para que la revista tenga una calidad incuestionable, es tener dos correctores, tanto de portugués como de inglés: uno que realice la primera corrección y otro que realice una segunda corrección, pero no tengo dinero para eso, entonces se hace lo que se puede. De vez en cuando encontramos algún error pero, en general, funciona bien. Todo el proceso de edición (corrección, diagramación e impresión) es un servicio que se contrata de forma tercerizada y tenemos a una persona que se dedica a coordinar ese proceso.

Como sistema de gestión, implementamos el programa ScholarOne, que si bien es de una empresa y lo paga SciELO Brasil, quien tiene contacto con nosotros es una persona muy buena, muy gentil, que está siempre trabajando con nosotros. Según Romeu Gomes (nuestro co-editor responsable, que realiza el seguimiento de los procesos de revisión) fue un hallazgo porque el programa ya tiene todo muy estandarizado. Lo que nosotros hicimos fue traducirlo al portugués, porque el programa estaba solo en inglés.

HS: ¿Y por qué no utilizan el Open Journal Systems (OJS)?

MCSM: No sabría decirte porqué. SciELO Brasil recomienda el programa ScholarOne para todas las revistas.

\section{HS: Pero el OJS es gratuito...}

MCSM: El ScholarOne también es gratuito para nosotros. Hay algunas cosas que teníamos anteriormente y que este programa no tiene. Por ejemplo, una vez que el artículo es aprobado, antes de realizar el proceso de edición y publicarlo dentro de un número, lo colocamos en la página web como "artículo en prensa", pero el programa no tiene esa posibilidad y tenemos que hacerlo por fuera del programa, de forma manual. Tenemos que rearmar el artículo para colocarlo en la página porque viene todo fragmentado del ScholarOne. Pero tiene otras ventajas. Por ejemplo, al mandar un artículo, hay un tiempo establecido, no sé si es un mes como máximo, y si el revisor no responde, el programa cierra la solicitud y pide que se asigne otro revisor. Ya no existe eso de que un artículo quede dando vueltas, esperando a que respondan. Tiene ventajas operativas que, según Romeu, son fantásticas. Es más complicado que el sistema que teníamos, constantemente necesitamos hacer capacitaciones, pero está yendo bien... A partir de ahora, el ScholarOne le va a asignar el digital object identifier (DOI) a cada artículo.

HS: Nosotros tenemos que pagar el DOI y Crossref nos cobra un dólar por artículo y una membrecía anual, y es un precio bajo por ser una revista editada por una universidad pública.

MCSM: Solo sé que nosotros nunca hemos pagado el DOI porque SciELO cubre ese gasto. Sin embargo, ahora tenemos que pagar otros servicios. En primer lugar, está el tema de la marcación $\mathrm{XML}$, que la realiza una empresa que está habilitada y brinda ese servicio a casi todas las revistas. Ese servicio lo pagamos, por ahora, con dinero de CNPq. La Fiocruz me prometió que iba a incluir el dinero necesario para la marcación dentro del monto general. Pero Fiocruz gasta solo en nuestra revista el $50 \%$ del presupuesto destinado a todas sus revistas, incluyendo Cadernos de Saúde Pública que publica 25 artículos por número, y Memórias do Instituto Oswaldo Cruz en la que los autores escriben diez páginas como máximo, un formato más característico de los biólogos y del campo biomédico. Tengo la promesa de Fiocruz de que ellos van a hacerse cargo del costo de la marcación XML.

Por otro lado, está el tema del inglés. Recibimos una presión muy fuerte de SciELO para publicar en inglés $y$, ¿por qué? Porque Ciência \& Saúde Coletiva es la revista brasileña -no solo del área de salud- que más artículos publica. Entonces, si publicara artículos en inglés, subiría el número de artículos en inglés del país y tuvimos una cierta presión. Entonces decidimos hacer un experimento: hice una carta que es una mezcla de cariño y presión [risas...], en la que le cuento al autor que SciELO está presionando cada 
vez más -no digo "presionando" sino "sugiriendo" - que las revistas brasileñas publiquen en inglés porque eso, según SciELO, aumenta el número de citas y la ciencia brasileña pasa a ser más leída, pero que ABRASCO no tiene dinero para financiarlo. Entonces sugerimos que, si la persona también cree que es importante, si le gustaría tener su artículo publicado en inglés y tiene condiciones de asumir el costo de la traducción, se contacte con alguno de los traductores que conocemos, y debajo incluimos los nombres de los traductores, porque no queremos que nos manden una traducción mal hecha. $Y$ también aclaro: "pero si su institución contara con un traductor oficial puede enviarnos la certificación". Percibí que una gran parte de los autores adhirieron y muchas personas decían: "Desgraciadamente no estaba enterada de esto, por lo que no tengo fondos para la traducción pero lo tendré en cuenta para la próxima vez". Es decir, la carta tuvo muy buena aceptación. Aunque siempre está aquel que dice "¿para qué hacer esto?". Hasta hoy, en las reuniones que tenemos con los editores asociados, hay cierta resistencia: "la traducción es muy difícil", "la traducción nunca es perfecta", "la calidad de las traducciones deja mucho que desear", todos esos comentarios están ahí en el área de salud pública. Sin embargo, no le doy mucha importancia porque a esta altura del partido quiero que esto se haga. Hice una estadística que llevé a la reunión del Congreso de ABRASCO, y desde diciembre hasta agosto teníamos el $62 \%$ de los artículos traducidos al inglés o escritos en inglés y la meta de SciELO es tener, al menos por ahora, el 50\%, por lo que nosotros ya alcanzamos la meta.

No tenés idea de lo que es dar cuenta de tantas cosas intentando pagar una cosa, la otra, la otra... Por ejemplo, la persona que hace la diagramación me reclama porque antes hacía la diagramación del portugués y ahora tiene que hacer también la del inglés y ¿quién paga eso? Yo le prometí que le pagaría 100 reales más por artículo, ¡me dedico a juntar dinero! No podés exigirles tanto sacrificio, las personas no tienen esa misma adhesión que uno tiene por la causa.
Esas exigencias de SciELO, tanto la traducción al inglés, como la marcación XML, que es para que un artículo sea leído a texto completo, son exigencias que hacen que la revista crezca, y la revista crece realmente. Yo formo parte de ResearchGate y soy una de las personas del área de la salud en un sentido amplio que tiene el mayor número de descargas de artículos, y veo cómo aumentó la lectura de mis textos desde que publico en inglés. Por ejemplo, en los últimos días tuve 34 lecturas en China, 28 de EE.UU., 8 en lengua española y 2 en lengua portuguesa, entonces no sé qué va a suceder a la hora de calcular el impacto, pero la realidad es que aumenta la visibilidad.

Este planteo ya estaba presente en la Conferencia "SciELO 15 años", pero en la reunión de los 16 años de SciELO la presión fue mayor, porque tanto Abel (Packer) como Meneghini fueron muy enfáticos en mostrar la necesidad de incorporar el inglés. Entonces el área de salud colectiva creó un Foro, por el que pasan todas las discusiones, en el que hay personas que plantean que no tienen dinero y que no les solicitarían a los autores que paguen la traducción, y otras que cuestionan que SciELO plantee eso como internacionalización. Yo fui criada por mi madre y por mi padre para obedecer y creo que tengo vocación militar [risas...] porque obedezco hasta el final. En la reunión mostré qué era lo que habíamos hecho: ampliamos el número de editores extranjeros del cuerpo editorial, si no recuerdo mal tenemos 22 personas. Y ¿cómo seleccionamos a esas personas? Contamos con un gran grupo de editores asociados que está conformado por áreas y les pedimos que nos indiquen personas que conozcan del exterior, que estén dispuestas a enviar trabajos, porque no queremos colocar solo el nombre de una persona sin que participe activamente. Entonces, aumentamos el número de investigadores extranjeros dentro del cuerpo editorial, logramos superar el $50 \%$ de artículos traducidos al inglés y hay un tercer aspecto, que es el más complicado, que es el número de autores extranjeros que publican en nuestras revistas. 
Cuando ellos hablan de autores extranjeros, casi siempre están hablando de autores de lengua inglesa. Entonces, qué intenté hacer y qué estoy haciendo. Actualmente los editoriales cuentan como producción científica, entonces, el editorial del número conmemorativo fue del presidente de la Federación Mundial de Asociaciones de Salud Pública, el anterior sobre tentativas de suicidio fue de Yeates Conwell que es un referente mundial en estudios de suicidio en adultos mayores. Estoy intentando hacer eso para aumentar la participación de autores extranjeros. Pero lo que señalé en un artículo que escribí sobre la revista ${ }^{(4)}$ es lo siguiente: en Brasil, por ejemplo, el financiamiento es muy bajo, y empeoró este año, y no solo de las revistas sino de las investigaciones. Entonces, ¿qué es lo que sucede? A pesar de todos los obstáculos, hay grupos de investigación que reciben financiamiento, pero solo hasta entregar el informe y no hay una política de publicación de resultados de investigación, que es algo caro para afrontar. En el caso de Brasil, CAPES paga no sé cuántos millones para que los investigadores accedan a las revistas extranjeras a través de su portal, pero no les paga nada a las revistas brasileñas que también están ahí. Es decir, para mantener, para crecer, para no quedar en ese nivel de voluntarismo, en el que uno se transforma casi en una ONG ambulante que sostiene una revista [risas...], se requiere financiamiento. A mi no me gusta quejarme, porque siempre intento resolverlo, sin atrasar nada, pero es realmente muy difícil. No sé cómo será aquí en Argentina, pero en Brasil se produjo una disminución de los fondos y lo que les dan a las revistas científicas es una insignificancia, una "merreca", no tengo otra palabra para definirlo. Por ejemplo, nuestra revista está entre las que más reciben, y nos entregan 50.000 reales por año. Con esa suma hago solo una edición y media, es lo que uso para pagarle a la persona que hace la diagramación, para pagarle a un pasante que nos ayuda, entonces, voy cubriendo los agujeros a medida que voy consiguiendo.
HS: ¿Cuál es el presupuesto que necesitarían?

MCSM: Si cuento la traducción al inglés, que para mí es lo más caro, necesitaría unos 50.000 reales por mes, para traducir los 30 artículos, sin contar los sueldos del personal. Si sumara los salarios ese monto se duplicaría.

VM: ¿Cuál es tu posición respecto a la categorización de Capes-Qualis?

MCSM: Cada área elabora sus propios parámetros de evaluación. Entonces, por ejemplo, un área que conozco bien, porque es mi área de origen, que es antropología, tiene cuatro revistas A1. Son revistas maravillosas pero muy poco citadas, son revistas importantísimas para el campo de la antropología y el área las categoriza como A1, y quien publica ahí, se lleva todos los méritos, porque eso repercute en la evaluación de los investigadores. En Brasil, el área de salud colectiva está dentro del área de medicina III, y los médicos del área parten del principio de que Brasil no tiene buenas revistas, de categoría, y ¿cuáles serían las revistas de categoría? Lancet, British Medical Journal, Science, etc., que en verdad tienen un factor de impacto muy alto, y nuestra revista nunca va a tener un factor de impacto similar, porque somos brasileños, no somos estadounidenses o ingleses. Y ellos categorizan como A1 solo a esas revistas que, además, la mayoría, son revistas médicas, no son revistas de salud pública y ahí es donde está la disputa política. Incluso Abel Packer me dijo: "No entiendo por qué Ciência \& Saúde Colectiva, Cadernos de Saúde Pública o Revista de Saúde Pública no son A1". Si esas tres revistas fueran A1, seguramente muchos de los que publican en el exterior publicarían en nuestras revistas. En todas las reuniones de ABRASCO, en todos los espacios que pudimos, planteamos este tema. Ahora está allí un profesor que se llama Guilherme Werneck, que es de la Universidade do Estado do Rio de Janeiro (UERJ), y creemos que posiblemente sea una persona con la que se pueda tener un mayor diálogo. Por ejemplo, Cadernos de Saúde Pública tiene un factor de impacto de 
0,70 y es $\mathrm{A} 2$, y la nuestra tiene 0,68 y es B1. La que tiene un factor de impacto un poco mayor es la Revista de Saúde Pública porque el crecimiento del factor de impacto está ligado a varios aspectos, entre ellos, el tiempo de la revista, el reconocimiento dentro de la comunidad académica, el respeto que la revista va adquiriendo para ser citada, para ser consultada, etc. Entonces, respecto a la categorización CAPES-Qualis diría lo siguiente: es un problema político, no es un problema de méritos, y es gracioso porque no es la política del Ministerio de Educación, es la política corporativa de un grupo.

VM: Pero si esto se lo vincula al escaso financiamiento que reciben las revistas brasileñas, ¿no se podría decir que hay una política que privilegia la publicación en revistas de la industria editorial por sobre las revistas brasileñas?

MCSM: Sí, es así, pero el año pasado CAPES, bajo la presidencia de Jorge Guimarães, decidió que, en función de todos los reclamos, iban a invertir en 100 revistas brasileñas para que pudieran crecer $y$, entre ellas, estaba la nuestra. Cada área tenía dos revistas, en nuestro caso creo que era la nuestra y Revista de Saúde Pública. Organizaron una reunión con Elsevier y una serie de empresas extranjeras y nos llamaron. Por un inconveniente de último momento, no pude asistir, ipor suerte!, porque en esa reunión se armó un gran alboroto al interior de la comunidad científica y los editores científicos: ¿por qué privilegiar solo a dos revistas por área?, ¿y las otras? El mundo de las revistas es ecosistémico, el crecimiento de una depende del crecimiento de las otras. En síntesis, CAPES canceló el proyecto, tuvo que cancelarlo porque la reacción de la comunidad fue muy fuerte, además de la reacción de SciELO, porque SciELO no fue invitado a participar de esa reunión. Como formo parte el consejo consultivo de SciELO Brasil, cuando recibí la invitación, le reenvié el correo a Abel y le pregunté: "¿estás al tanto de esta reunión?", y él me respondió: "Cecília, yo me enteré por otras personas, pero quería decirte que es una forma de boicotear a SciELO". Yo no sé si es verdad o no, porque SciELO en Brasil actualmente es casi propiedad de la Fundação de Amparo à Pesquisa do Estado de São Paulo (FAPESP), el $90 \%$ del presupuesto es de FAPESP y solo el 10\% es de Capes y CNPq. Algo que debería ser nacional está siendo capitaneado por una institución de investigación paulista, que ya tiene tendencia a dominar... pero igracias a Dios! ¡Viva FAPESP! [risas...]

VM: En Brasil, siempre tuvieron un diálogo fluido entre editores.

MCSM: Dentro del área de salud pública, sí.

VM: ¿Pudieron construir juntos, hay cosas que construyeron juntos?

MCSM: Lo que vamos construyendo juntos es un consenso. Por ejemplo, considero que SciELO es un proyecto nacional de Brasil, entonces no es un enemigo, no me voy a pelear con SciELO. Pero últimamente las reuniones han sido para discutir las demandas de SciELO y para plasmar por escrito los desacuerdos. En la página de $A B R A S C O$ se puede encontrar un documento del Foro de editores, en el que se menciona la disconformidad con relación a las exigencias de SciELO. Y yo fui clara, y dije: "voy a firmar y a apoyar el documento que surja del grupo, pero lo que SciELO está intentando es ver cómo puede aumentar la producción científica brasileña y provocar más impacto", que es algo que debería hacer el Ministerio de Ciencia y Tecnología.

VM: Para cerrar, ¿Cuál es tu balance de la experiencia como editora de Ciência \& Saúde Coletiva?

MCSM: Estoy muy orgullosa de la revista, de lo que se hizo, del equipo, es un equipo maravilloso que se pone la camiseta, que gana poco y trabaja mucho. Y creo que más allá de mi visión personal, desde el punto de vista de la representación, nuestra revista ha estado presente en todos los grandes eventos de salud pública del país. En Brasil tenemos el Instituto Brasileño de Geografía y Estadística 
(IBGE), que cada cuatro años hace la Encuesta Nacional de Salud, y nosotros publicamos el análisis de los resultados. Estuvimos presentes en Río + 20, es decir, estamos presentes en todos los eventos relevantes de salud pública y de publicación científica del país. Hicimos un balance en un artículo reciente ${ }^{(4)}$, en el que mostramos que es la revista que más publica sobre políticas de salud del país, que más publica sobre atención básica en salud, es decir, hay una cantidad de temas en los que estamos en primer lugar $y$, si no me equivoco, creo que el $23 \%$ de la publicación científica dentro del área de salud pública es de la revista. Y en las métricas de citación de Google Scholar, entre las revistas brasileñas de todas las áreas de conocimiento, ocupamos el primer lugar durante dos años consecutivos. Creo que es para estar orgullosa, ¿no? De todos modos, a mí no me gusta mucho mirar hacia atrás. Lo que puedo decir es que la salida de cada número es un acontecimiento, es una dificultad, una trama compleja, sobre todo para quien está haciendo el trabajo más sistemático. Es difícil, pero nosotros estamos siempre al día con la revista y ¡cada edición es una fiesta!

HS: ¿Alguna vez imaginaste que ibas a sacar un número por mes?

MCSM: Nunca, y mucho menos con treinta artículos [risas...].

VM: Fue un enorme placer...

HS: Muito obrigado!

\section{REFERENCIAS BIBLIOGRÁFICAS}

1. Ciência \& Saúde Coletiva [Internet]. 2016 [citado 10 jul 2016]. Disponible en: http://www. cienciaesaudecoletiva.com.br

2. Ciência \& Saúde Coletiva [Internet]. SciELO Brasil; 2016 [citado 10 jul 2016]. Disponible en: http://goo.gl/SOEMMT.

3. Almeida Filho N. Transdisciplinaridade e Saúde Coletiva. Ciência \& Saúde Coletiva. 1997;2(12):5-20.
4. Minayo MCS, Gomes R. Ciência \& Saúde Coletiva no contexto nacional e internacional da divulgação científica. Ciência \& Saúde Coletiva. 2015;20(7):2013-2022.

\section{NOTAS FINALES}

[a] Hace referencia al artículo de Naomar de Almeida Filho "Transdisciplinaridade e Saúde Coletiva"(1) que da origen a un debate del que participaron José da Rocha Carvalheiro, José Ricardo de Carvalho Mesquita Ayres, Luis David Castiel, entre otros. 